\title{
TWISTING OF SIEGEL MODULAR FORMS WITH CHARACTERS, AND L-FUNCTIONS
}

\author{
A. ANDRIANOV
}

\begin{abstract}
Linear twistings of Siegel modular forms with Dirichlet characters are considered. It is shown that the twisting operators transform modular forms to modular forms. Commutation of twisting operators and Hecke operators is examined. It is proved that under certain conditions the spinor zeta-function of a twisted modular form can be interpreted as the $L$-function of the initial modular form with twisting character. As an illustration of the twist techniques, analytic properties of $L$-functions of cusp forms of genus $n=1$ are considered.
\end{abstract}

\section{INTRODUCTION}

Consider a function, say, a Siegel modular form of genus $n \geq 1$, given on the upper half-plane

$$
\mathbb{H}^{n}=\left\{Z=X+i Y \in \mathbb{C}_{n}^{n} \mid{ }^{t} Z=Z, Y>0 \quad(i=\sqrt{-1})\right\}
$$

by an absolutely convergent Fourier series of the form

$$
F(Z)=\sum_{A \in \mathbb{A}^{n}, A \geq 0} f(A) e^{2 \pi i \operatorname{Tr}(A Z)}
$$

with constant Fourier coefficients $f(A)$, where the series is assumed to converge uniformly on compact sets, and $A$ ranges over all positive semidefinite matrices belonging to the set

$$
\mathbb{A}^{n}=\left\{A=\left(a_{\alpha \beta}\right) \in \frac{1}{2} \mathbb{Z}_{n}^{n} \mid{ }^{t} A=A, \quad a_{11}, a_{22}, \ldots, a_{n n} \in \mathbb{Z}\right\}
$$

of all symmetric half-integer matrices of order $n$ with integral entries on the principal diagonal. Investigation of Euler products corresponding to modular forms leads to the natural question, which was once proposed by A. A. Panchishkin, on twisting the series (1) with multiplicative functions such as characters. This paper is an attempt to address this question.

If $n=1$, then it is natural to define the twisting of a Fourier series

$$
\sum_{a \in \mathbb{Z}, a \geq 0} f(a) e^{2 \pi i a z}
$$

with a character $\chi$ by the Fourier series

$$
\sum_{a \in \mathbb{Z}, a \geq 0} \chi(a) f(a) e^{2 \pi i a z} .
$$

But if $n>1$, then, while considering linear twistings, i.e., such that a character of a linear form in coefficients of the running matrix $A \in \mathbb{A}^{n}$ is involved, there is no a priori

2000 Mathematics Subject Classification. Primary 11F46, 11F60, 11F66.

Key words and phrases. Hecke operators, Siegel modular forms, zeta-functions of modular forms.

Supported in part by RFBR (grant no. 08-01-00233). 
reason to prefer one linear form to another. Therefore, it is quite natural to consider twistings with characters of arbitrary integral linear forms in coefficients of the matrix $A$. Obviously, every such form can be written as $L(A)=\operatorname{Tr}(L A)$ with an integral symmetric matrix

$$
L={ }^{t} L \in \mathbb{Z}_{n}^{n}
$$

of order $n$. Thus, we get the twisting of Fourier series $F$ with a character $\chi$ and a p-matrix (parameter-matrix) $L$; this twisting is defined by the series

$$
(F \mid \mathcal{T}(\chi, L))(Z)=\sum_{A \in \mathbb{A}^{n}, A \geq 0} \chi(\operatorname{Tr}(L A)) f(A) e^{2 \pi i \operatorname{Tr}(A Z)} \quad\left(Z \in \mathbb{H}^{n}\right) .
$$

The operator

$$
|\mathcal{T}(\chi, L): F \mapsto F| \mathcal{T}(\chi, L)
$$

will be called the twisting operator with character $\chi$ and p-matrix $L$.

Zeta-functions and $L$-functions of modular forms of genus $n=1$ (elliptic modular forms) were investigated by Erich Hecke and his followers. In particular, it was proved that these functions have meromorphic analytic continuation to the entire $s$-plane and satisfy a functional equation (see, e.g., [6, Chapters 1 and 5]). Analytic continuation to the entire $s$-plane and a functional equation for the zeta-function of Siegel modular forms of genus $n=2$ for the full modular group $\Gamma^{2}=S p_{2}(\mathbb{Z})$ were proved in [2] and [3]. A reasonably full investigation of zeta-functions and $L$-functions of modular forms of genus $n>2$ is a problem for the distant future. Here we make an initial step in this direction.

Contents. In $\S \S 1-3$ we consider the action of twisting operators on Siegel modular forms of an arbitrary genus $n \geq 1$. In particular, in $\S 1$ we show that, under certain assumptions, the twisting operators take modular forms to modular forms. In $\S 2$ we treat commutation relations between twisting operators and Hecke operators. In $\S 3$ we prove that, under certain conditions, the zeta-function of a twisted modular form can be interpreted as the $L$-function of the initial modular form with the character of twisting. As an illustration, in $\S 4$ we show how the twisting techniques can be used to investigate analytic properties of $L$-functions of cusp forms of genus $n=1$, viewed as zeta-functions of twisted forms. In forthcoming papers we intend to apply this approach to the investigation of $L$-functions of modular forms of genus $n=2$.

Notation. The letters $\mathbb{N}, \mathbb{Z}, \mathbb{Q}, \mathbb{R}$, and $\mathbb{C}$ are reserved for the set of positive rational integers, the ring of rational integers, the field of rational numbers, the field of real numbers, and the field of complex numbers, respectively.

$\mathbb{X}_{n}^{m}$ is the set of all $(m \times n)$-matrices with entries in a set $\mathbb{X}$. If $M$ is a matrix, ${ }^{t} M$ always denotes the transpose of $M$, and for a square matrix $M, \operatorname{Tr}(M)$ is the sum of diagonal elements of $M$. If $Y$ is a real symmetric matrix, then $Y>0$ (respectively, $Y \geq 0$ ) means that $Y$ is positive definite (respectively, positive semidefinite). $1_{n}=\mathbf{1}$ and $0_{n}=\mathbf{0}$ denote the unit matrix and the zero matrix of order $n$, respectively. The bar over a complex number or character means complex conjugation.

We use the notation

$$
A[B]={ }^{t} B A B
$$

for two matrices of suitable dimension, and the abbreviation

$$
\langle n\rangle=n(n+1) / 2 .
$$




\section{$\S 1$. TWisting OF MODUlar FORMS With CHARACTERS}

In this section we show, in particular, that under certain conditions the twisting operators take modular forms to modular forms, and we examine the corresponding groups.

Let

$$
\mathbb{G}^{n}=\left\{M \in \mathbb{R}_{2 n}^{2 n} \mid{ }^{t} M J_{n} M=\mu(M) J_{n}, \quad \mu(M)>0\right\} \quad \text { with } \quad J_{n}=\left(\begin{array}{cc}
0_{n} & 1_{n} \\
-1_{n} & 0_{n}
\end{array}\right)
$$

be the connected component of the unit in the general real symplectic group of genus $n$. The group $\mathbb{G}^{n}$ acts as a group of analytic automorphisms of the upper half-plane $\mathbb{H}^{n}$ by the rule

$$
\mathbb{G}^{n} \ni M=\left(\begin{array}{ll}
A & B \\
C & D
\end{array}\right): Z \mapsto M\langle Z\rangle=(A Z+B)(C Z+D)^{-1} \quad\left(Z \in \mathbb{H}^{n}\right) .
$$

Acting on the upper half-plane, the group $\mathbb{G}^{n}$ operates on functions $F: \mathbb{H}^{n} \mapsto \mathbb{C}$ by (normalized) Petersson operators of integral weights $k$,

$$
\begin{aligned}
\mathbb{G}^{n} \ni M & =\left(\begin{array}{ll}
A & B \\
C & D
\end{array}\right):\left.F \mapsto F\right|_{k} M \\
& =\mu(M)^{n k-\langle n\rangle} \operatorname{det}(C Z+D)^{-k} F(M\langle Z\rangle)
\end{aligned}
$$

(see (6)). The normalizing factor $\mu(M)^{n k-\langle n\rangle}$, going back for $n=1$ to Hecke, is not significant by itself, but it simplifies a number of formulas related to Hecke operators. The Petersson operators transform isomorphically the space of functions on $\mathbb{H}^{n}$ into itself, map holomorphic functions to holomorphic functions, and satisfy the rule

$$
\left.F\right|_{k} M M^{\prime}=\left.\left.F\right|_{k} M\right|_{k} M^{\prime} \text { for all } M, M^{\prime} \in \mathbb{G}^{n}
$$

(see, e.g., [4, Lemmas 1.4.1, 1.4.2]).

In the above notation, the following statement is true.

Lemma 1.1. If $F$ is a function on $\mathbb{H}^{n}$ with Fourier expansion (1), $\chi$ is a Dirichlet character modulo $m \in \mathbb{N}$, and $L$ is a matrix of the form (2), then the twisting of $F$ with character $\chi$ and p-matrix $L$ can be written with the help of the Petersson operators (1.1) of an arbitrary integral weight $k$ in the form

$$
F\left|\mathcal{T}(\chi, L)=\frac{1}{m} \sum_{r, l \bmod m} \chi(r) e^{-\frac{2 \pi i r l}{m}} F\right|_{k} U\left(m^{-1} l L\right)
$$

where, for a real symmetric matrix $B$ of order $n$, we use the notation

$$
U(B)=\left(\begin{array}{cc}
\mathbf{1} & B \\
\mathbf{0} & \mathbf{1}
\end{array}\right) \in \mathbb{G}^{n}
$$

Proof. The definitions and the Fourier expansion (1) imply that

$$
\left(F \mid{ }_{k} U\left(m^{-1} l L\right)\right)(Z)=F\left(Z+m^{-1} l L\right)=\sum_{A \in \mathbb{A}, A \geq 0} f(A) e^{\frac{2 \pi i l}{m} \operatorname{Tr}(L A)} e^{2 \pi i \operatorname{Tr}(A Z)} .
$$


Consequently,

$$
\begin{aligned}
\left.\frac{1}{m} \sum_{r, l \bmod m} \chi(r) e^{-\frac{2 \pi i r l}{m}} F\right|_{k} U\left(m^{-1} l L\right) \\
\quad=\frac{1}{m} \sum_{r, l \bmod m} \chi(r) e^{-\frac{2 \pi i r l}{m}} \sum_{A \in \mathbb{A}, A \geq 0} f(A) e^{\frac{2 \pi i l}{m} \operatorname{Tr}(L A)} e^{2 \pi i \operatorname{Tr}(A Z)} \\
=\sum_{r \bmod m} \chi(r) \sum_{A \in \mathbb{A}, A \geq 0} \frac{1}{m} \sum_{l \bmod m} e^{\frac{2 \pi i l}{m}(-r+\operatorname{Tr}(L A))} f(A) e^{2 \pi i \operatorname{Tr}(A Z)} \\
=\sum_{A \in \mathbb{A}, A \geq 0} \chi(\operatorname{Tr}(L A)) f(A) e^{2 \pi i \operatorname{Tr}(A Z)}=(F \mid \mathcal{T}(\chi, L))(Z) .
\end{aligned}
$$

For a Dirichlet character $\chi$ modulo $m \in \mathbb{N}$ and an integer $l \in \mathbb{Z}$, we introduce the Gaussian sums

$$
g(\chi, l)=\sum_{r \bmod m} \chi(r) e^{\frac{2 \pi i l r}{m}} \text { and } g(\chi)=g(\chi, 1) .
$$

The character $\chi$ is called a primitive character modulo $m$ if it is a character modulo $m$, but not modulo any proper divisor of $m$. If $\chi$ is a primitive character modulo $m$, then the Gaussian sums satisfy the relations

$$
g(\chi, r)=\bar{\chi}(r) g(\chi), \quad|g(\chi)|=\sqrt{m}
$$

(see, e.g., [6, Proposition 21]).

Lemma 1.2. In the notation of the preceding lemma, if, moreover, the character $\chi$ is primitive modulo $m$, then formula (1.3) can be written in the form

$$
F\left|\mathcal{T}(\chi, L)=\frac{g(\chi)}{m} \sum_{l \bmod m} \bar{\chi}(-l) F\right|_{k} U\left(m^{-1} l L\right),
$$

where $g(\chi)=g(\chi, 1)$ is the Gaussian sum and $\bar{\chi}$ is the complex conjugate of the character $\chi$.

Proof. If $\chi$ is primitive, then, by Lemma 1.1 and (1.5),

$$
\begin{aligned}
F \mid \mathcal{T}(\chi, L) & =\left.\frac{1}{m} \sum_{l \bmod m}\left(\sum_{r \bmod m} \chi(r) e^{-\frac{2 \pi i r l}{m}}\right) F\right|_{k} U\left(m^{-1} l L\right) \\
& =\left.\frac{1}{m} \sum_{l \bmod m} \bar{\chi}(-l) g(\chi) F\right|_{k} U\left(m^{-1} l L\right) .
\end{aligned}
$$

We recall that a (holomorphic) modular form of genus $n \geq 1$ and integral weight $k$ for a subgroup $\Lambda$ of finite index in the modular group $S p_{n}(\mathbb{Z})$ of genus $n$ is defined as a holomorphic function $F$ on $\mathbb{H}^{n}$ that satisfies

$$
\left.F\right|_{k} M=F \quad \text { for each } \quad M \in \Lambda,
$$

and, if $n=1$, is regular at all cusps of $\Lambda$. All such functions form a finite-dimensional linear space $\mathfrak{M}_{k}(\Lambda)$ over $\mathbb{C}$. The forms equal to zero at all cusps are called cusp forms; they constitute the subspace of cusp forms $\mathfrak{N}_{k}(\Lambda)$.

In this paper we consider modular forms for the full modular group

$$
\Gamma=\Gamma^{n}=S p_{n}(\mathbb{Z})=\left\{M \in \mathbb{G}^{n} \cap \mathbb{Z}_{2 n}^{2 n} \mid \mu(M)=1\right\},
$$

for congruence subgroups of type

$$
\Gamma_{0}(m)=\Gamma_{0}^{n}(m)=\left\{M=\left(\begin{array}{cc}
A & B \\
C & D
\end{array}\right) \in \Gamma \mid C \equiv \mathbf{0} \quad(\bmod m)\right\}
$$


and

$$
\Gamma_{[0]}(m)=\Gamma_{[0]}^{n}(m)=\left\{M=\left(\begin{array}{ll}
A & B \\
C & D
\end{array}\right) \in \Gamma_{0}(m) \mid B \equiv \mathbf{0} \quad(\bmod m)\right\},
$$

for the principal congruence subgroup of level $m$,

$$
\Gamma(m)=\Gamma^{n}(m)=\left\{M \in \Gamma^{n} \mid M \equiv 1_{2 n} \quad(\bmod m)\right\},
$$

and for subgroups conjugate to $\Gamma_{[0]}(m)$ and $\Gamma(m)$,

$$
\widetilde{\Gamma}_{[0]}(m)=\widetilde{\Gamma}_{[0]}^{n}(m)=V(m) \Gamma_{[0]}(m) V(m)^{-1}=\Gamma_{0}\left(m^{2}\right)
$$

and

$$
\begin{aligned}
\widetilde{\Gamma}(m) & =\widetilde{\Gamma}^{n}(m)=V(m) \Gamma(m) V(m)^{-1} \\
& =\left\{\left(\begin{array}{ll}
A & B \\
C & D
\end{array}\right) \in \Gamma \mid A \equiv D \equiv \mathbf{1} \quad(\bmod m), C \equiv \mathbf{0} \quad\left(\bmod m^{2}\right)\right\},
\end{aligned}
$$

where, for fixed $n, m \geq 1$, we set

$$
V(m)=V^{n}(m)=\left(\begin{array}{cc}
\mathbf{1} & \mathbf{0} \\
\mathbf{0} & m \mathbf{1}
\end{array}\right) .
$$

We shall consider the spaces $\mathfrak{M}_{k}(\Lambda)$ of all modular forms and the spaces $\mathfrak{N}_{k}(\Lambda)$ of all cusp forms of a fixed integral weight $k$ for the groups $\Lambda$ introduced above. In the following lemma, we list useful properties of the matrices (1.4) and the corresponding Petersson operators.

Lemma 1.3. 1) Every matrix $M=(\underset{C}{A} \stackrel{B}{D})$ of order $2 n$ with blocks of order $n$ and arbitrary matrices of the form (1.4) satisfy the relation

$$
U\left(B^{\prime}\right)\left(\begin{array}{ll}
A & B \\
C & D
\end{array}\right) U\left(B^{\prime \prime}\right)^{-1}=\left(\begin{array}{cc}
A+B^{\prime} C & B^{\prime} D-A B^{\prime \prime}+B-B^{\prime} C B^{\prime \prime} \\
C & -C B^{\prime \prime}+D
\end{array}\right) .
$$

2) For every matrix of the form (1.4) with $B=m^{-1} L$, where $L$ is as in (2), the group $\widetilde{\Gamma}(m)$ satisfies the relation

$$
U\left(m^{-1} L\right) \widetilde{\Gamma}(m) U\left(m^{-1} L\right)^{-1}=\widetilde{\Gamma}(m) .
$$

3) The operators $\left.\right|_{k} U\left(m^{-1} L\right)$ with $L$ as in $(2)$ map the spaces $\mathfrak{M}_{k}(\widetilde{\Gamma}(m))$ and $\mathfrak{N}_{k}(\widetilde{\Gamma}(m))$ onto themselves.

Proof. Formula (1.13) is a result of multiplication of block matrices. By this formula, for $M=\left(\begin{array}{cc}A & B \\ C & D\end{array}\right) \in \widetilde{\Gamma}(m)$, we obtain the matrix

$$
\begin{aligned}
U\left(m^{-1} L\right) & \left(\begin{array}{cc}
A & B \\
C & D
\end{array}\right) U\left(m^{-1} L\right)^{-1} \\
= & \left(\begin{array}{cc}
A+m^{-1} L C & m^{-1}(L D-A L)+B-m^{-2} L C L \\
C & -m^{-1} C L+D
\end{array}\right),
\end{aligned}
$$

which, obviously, belongs to $\widetilde{\Gamma}(m)$. This proves that the left-hand side of (1.14) is contained in the right-hand side. The reverse inclusion follows similarly, because, clearly, $U(B)^{-1}=U(-B)$.

Finally, to prove statement 3 ), actually it suffices to check, e.g., that for the function $F^{\prime}=\left.F\right|_{k} U\left(m^{-1} L\right)$ with $F \in \mathfrak{M}_{k}(\widetilde{\Gamma}(m))$ we have $\left.F^{\prime}\right|_{k} M=F^{\prime}$ for all $M \in \widetilde{\Gamma}(m)$. By $(1.2)$,

$$
\left.F^{\prime}\right|_{k} M=\left.F\right|_{k} U\left(m^{-1} L\right) M=\left.\left.F\right|_{k} U\left(m^{-1} L\right) M U\left(m^{-1} L\right)^{-1}\right|_{k} U\left(m^{-1} L\right)=F^{\prime},
$$

because $U\left(m^{-1} L\right) M U\left(m^{-1} L\right)^{-1} \in \widetilde{\Gamma}(m)$ by (1.14). 
Proposition 1.4. For every Dirichlet character $\chi$ modulo $m$ and every p-matrix $L$ of the form (2), the twisting operator $\mid \mathcal{T}(\chi, L)$ maps the spaces $\mathfrak{M}_{k}(\widetilde{\Gamma}(m))$ and $\mathfrak{N}_{k}(\widetilde{\Gamma}(m))$ of modular forms and cusp forms of weight $k$ for the group $\widetilde{\Gamma}(m)$ into themselves.

Proof. Note that every function in these spaces has a Fourier expansion of the form (1). The assertion directly follows from formula (1.3) and statement 3) of Lemma 1.3 with $l L$ in place of $L$.

Proposition 1.5. If $F \in \mathfrak{M}_{k}\left(\Gamma_{0}\left(m^{2}\right)\right), \chi$ is a character modulo $m$, and $L$ is a matrix of the form (2), then the twist of the form $F$ with character $\chi$ and p-matrix $L$ satisfies the rules

$$
\left.(F \mid \mathcal{T}(\chi, L))\right|_{k}\left(\begin{array}{ll}
A & B \\
C & D
\end{array}\right)=F \mid \mathcal{T}(\chi, L[D]), \quad\left(\begin{array}{ll}
A & B \\
C & D
\end{array}\right) \in \Gamma_{0}\left(m^{2}\right),
$$

where $L[D]={ }^{t} D L D$. If, moreover, the block $D$ satisfies the congruence

$$
L[D] \equiv \nu(D) L \quad(\bmod m) \quad \text { with a scalar } \quad \nu(D)=\nu_{L}(D),
$$

then for the function $F \mid \mathcal{T}(\chi, L)$ we have

$$
\left.(F \mid \mathcal{T}(\chi, L))\right|_{k}\left(\begin{array}{ll}
A & B \\
C & D
\end{array}\right)=\chi(\nu(D)) F \mid \mathcal{T}(\chi, L) .
$$

Proof. If $M=\left(\begin{array}{cc}A & B \\ C\end{array}\right) \in \Gamma_{0}\left(m^{2}\right)$ and $l \in \mathbb{Z}$, then, by (1.3) and (1.2), we can write

$$
\begin{aligned}
& \left.(F \mid \mathcal{T}(\chi, L))\right|_{k} M=\left.\left.\frac{1}{m} \sum_{r, l \bmod m} \chi(r) e^{-\frac{2 \pi i r l}{m}} F\right|_{k} U\left(m^{-1} l L\right)\right|_{k} M \\
& \quad=\left.\frac{1}{m} \sum_{r, l \bmod m} \chi(r) e^{-\frac{2 \pi i r l}{m}} F\right|_{k} U\left(m^{-1} l L\right) M U\left(m^{-1} l L[D]\right)^{-1} U\left(m^{-1} l L[D]\right) \\
& \quad=\left.\left.\frac{1}{m} \sum_{r, l \bmod m} \chi(r) e^{-\frac{2 \pi i r l}{m}} F\right|_{k} M_{l}^{\prime}\right|_{k} U\left(m^{-1} l L[D]\right),
\end{aligned}
$$

where $M_{l}^{\prime}=U\left(m^{-1} l L\right) M U\left(m^{-1} l^{t} D L D\right)^{-1}$. By statement 1$)$ of Lemma 1.3 with $B^{\prime}=$ $m^{-1} l L$ and $B^{\prime \prime}=m^{-1} l L[D]$, we obtain

$$
\begin{aligned}
M_{l}^{\prime} & =\left(\begin{array}{cc}
A+m^{-1} l L C & m^{-1} l(L D-A L[D])+B-m^{-2} l^{2} L C L[D] \\
C & -m^{-1} l C L[D]+D
\end{array}\right) \\
& =\left(\begin{array}{cc}
A+m^{-1} l L C & -m^{-1} l\left(-A^{t} D+1_{n}\right) L D+B-m^{-2} l^{2} L C C^{t} D L D \\
C & -m^{-1} l C C^{t} D L D+D
\end{array}\right) .
\end{aligned}
$$

Clearly, this matrix is integral. Moreover, it belongs to the group $\mathbb{G}^{n}$, and satisfies $\mu\left(M_{l}^{\prime}\right)=\mu(M)=1$. Since $C \equiv \mathbf{0}\left(\bmod m^{2}\right)$, it follows that $M_{l}^{\prime} \in \Gamma_{0}\left(m^{2}\right)$. Hence, $\left.F\right|_{k} M_{l}^{\prime}=F$, and applying formula (1.3) once again, we get

$$
\left.(F \mid \mathcal{T}(\chi, L))\right|_{k} M=\left.\frac{1}{m} \sum_{r, l \bmod m} \chi(r) e^{-\frac{2 \pi i r l}{m}} F\right|_{k} U\left(m^{-1} l L[D]\right)=F \mid \mathcal{T}(\chi, L[D]) .
$$

Turning to (1.17), we use (1.15) to obtain

$$
\begin{aligned}
\left.(F \mid \mathcal{T}(\chi, L))\right|_{k}\left(\begin{array}{ll}
A & B \\
C & D
\end{array}\right) & =\sum_{A \in \mathbb{A}, A \geq 0} \chi(\operatorname{Tr}(L[D] A)) f(A) e^{2 \pi i(\operatorname{Tr}(A Z))} \\
& =\sum_{A \in \mathbb{A}, A \geq 0} \chi\left(\nu(D)(\operatorname{Tr}(L A)) f(A) e^{2 \pi i(\operatorname{Tr}(A Z))}\right. \\
& =\chi(\nu(D)) F \mid \mathcal{T}(\chi, L) .
\end{aligned}
$$


Lemma 3.3.2(1) in [4 implies that the natural mapping of the group $\Gamma_{0}\left(m^{2}\right)$ to the group $G L_{n}(\mathbb{Z} / m \mathbb{Z})$ of nonsingular matrices of order $n$ over the residue ring $\mathbb{Z} / m \mathbb{Z}$ given by

$$
\Gamma_{0}\left(m^{2}\right) \ni M=\left(\begin{array}{ll}
A & B \\
C & D
\end{array}\right) \mapsto D / \bmod m \in G L_{n}(\mathbb{Z} / m \mathbb{Z})
$$

is an epimorphic homomorphism with the kernel $\widetilde{\Gamma}(m)$. For a matrix $D$ in the group $G L_{n}(\mathbb{Z} / m \mathbb{Z})$, we denote by

$$
\varrho(D) \in \Gamma_{0}\left(m^{2}\right)
$$

some inverse image of $D$ under this mapping. Since $\widetilde{\Gamma}(m)$ is a normal subgroup of $\Gamma_{0}\left(m^{2}\right)$, the cosets

$$
\widetilde{\Gamma}(m) \varrho(D)=\varrho(D) \widetilde{\Gamma}(m)=\widetilde{\Gamma}(m) \varrho(D) \widetilde{\Gamma}(m)
$$

are independent of the choice of $D \in G L_{n}(\mathbb{Z} / m \mathbb{Z})$. Hence, each operator

$$
\left.\right|_{k} \varrho(D):\left.F \mapsto F\right|_{k} \varrho(D) \quad\left(D \in G L_{n}(\mathbb{Z} / m \mathbb{Z}), F \in \mathfrak{M}_{k}(\widetilde{\Gamma}(m))\right)
$$

depends only on the matrix $D$ modulo $m$ and maps the space $\mathfrak{M}_{k}(\widetilde{\Gamma}(m))$ onto itself. Moreover, these operators satisfy

$$
\left.\left.\right|_{k} \varrho(D)\right|_{k} \varrho\left(D^{\prime}\right)=\left.\right|_{k} \varrho\left(D D^{\prime}\right) \quad\left(D, D^{\prime} \in G L_{n}(\mathbb{Z} / m \mathbb{Z})\right) .
$$

Now, Proposition 1.5 can be reformulated in terms of the operators $\left.\right|_{k} \varrho(D)$.

Proposition 1.6. If $F \in \mathfrak{M}_{k}\left(\Gamma_{0}\left(m^{2}\right)\right)$, then

$$
\left.(F \mid \mathcal{T}(\chi, L))\right|_{k} \varrho(D)=F \mid \mathcal{T}(\chi, L[D]) \quad \text { for all } \quad D \in G L_{n}(\mathbb{Z} / m \mathbb{Z}) .
$$

If, moreover, the matrix $D$ satisfies (1.16), then this formula turns into

$$
\left.(F \mid \mathcal{T}(\chi, L))\right|_{k} \varrho(D)=\chi(\nu(D)) F \mid \mathcal{T}(\chi, L) .
$$

In particular,

$$
\left.(F \mid \mathcal{T}(\chi, L))\right|_{k} \tau(d)=\chi\left(d^{2}\right) F \mid \mathcal{T}(\chi, L) \quad \text { for all } d \in \mathbb{N} \text { prime to } m,
$$

where

$$
\tau(d)=\tau^{n}(d)=\varrho\left(d \cdot 1_{n}\right) .
$$

Along with operators (1.20), we shall also use the Petersson operators corresponding to matrices of order $2 n$ of the form

$$
\Omega=\Omega^{n}(m)=\left(\begin{array}{cc}
\mathbf{0} & m^{-1} \mathbf{1} \\
-m \mathbf{1} & \mathbf{0}
\end{array}\right) .
$$

It is easily seen that $\Omega \in \mathbb{G}^{n}, \mu(\Omega)=1$,

$$
\Omega^{-1}=-\Omega, \text { and } \quad \Omega^{2}=-1_{2 n} .
$$

The obvious relations

$$
\Omega\left(\begin{array}{ll}
A & B \\
C & D
\end{array}\right) \Omega^{-1}=\Omega^{-1}\left(\begin{array}{ll}
A & B \\
C & D
\end{array}\right) \Omega=\left(\begin{array}{cc}
D & -\frac{C}{m^{2}} \\
-m^{2} B & A
\end{array}\right)
$$

valid for every $2 n$-matrix $\left(\begin{array}{cc}A & B \\ C & D\end{array}\right)$ with $n$-blocks $A, B, C, D$, and the definitions of the groups $\Lambda=\Gamma_{0}\left(m^{2}\right)$ and $\widetilde{\Gamma}(m)$ show that for these groups we have

$$
\Omega \Lambda \Omega^{-1}=\Omega^{-1} \Lambda \Omega=\Lambda .
$$

Coming back to modular forms, we have the following assertions. 
Proposition 1.7. 1) In the notation (1.1) and (1.25), the linear operator

$$
\left.\right|_{k} \Omega: F \mapsto F^{*}=\left.F\right|_{k} \Omega
$$

maps the spaces $\left.\mathfrak{M}_{k}\left(\Gamma_{0}\left(m^{2}\right)\right), \mathfrak{M}_{k}(\widetilde{\Gamma}(m))\right), \mathfrak{N}_{k}\left(\Gamma_{0}\left(m^{2}\right)\right)$, and $\mathfrak{N}_{k}(\widetilde{\Gamma}(m))$ onto themselves, and

$$
\left(F^{*}\right)^{*}=(-1)^{k} F .
$$

2) The operators $(1.29)$ and (1.20) on the spaces $\mathfrak{M}_{k}(\widetilde{\Gamma}(m))$ and $\mathfrak{N}_{k}(\widetilde{\Gamma}(m)$ ) (respectively) satisfy the relation

$$
\left|{ }_{k} \Omega\right|_{k} \varrho(D)=\left.{ }_{k} \varrho\left({ }^{t} D^{-1}\right)\right|_{k} \Omega,
$$

where $D^{-1}$ is the inverse of $D$ modulo $m$.

3) In the notation of Proposition 1.4, we have

$$
\left.(F \mid \mathcal{T}(\chi, L))^{*}\right|_{k} \varrho(D)=\left(F \mid \mathcal{T}\left(\chi, L\left[{ }^{t} D^{-1}\right]\right)\right)^{*} ;
$$

if, moreover, the matrix $D$ satisfies (1.16), then formula (1.32) turns into

$$
\left.(F \mid \mathcal{T}(\chi, L))^{*}\right|_{k} \varrho(D)=\bar{\chi}(\nu(D))(F \mid \mathcal{T}(\chi, L))^{*} ;
$$

in particular,

$$
(F \mid \mathcal{T}(\chi, L))^{*}{ }_{k} \tau(d)=\bar{\chi}\left(d^{2}\right)(F \mid \mathcal{T}(\chi, L))^{*}
$$

for all $d \in \mathbb{N}$ prime to $m$, where $\tau(d)$ denotes a matrix of the form (1.24).

Proof. Using (1.29), (1.28), and (1.2), for $M \in \Lambda=\Gamma_{0}\left(m^{2}\right)$ or $\widetilde{\Gamma}(m)$ and $F \in \mathfrak{M}_{k}(\Lambda)$ or $\mathfrak{N}_{k}(\Lambda)$, we get

$$
\left.F^{*}\right|_{k} M=\left.F\right|_{k} \Omega M=\left.F\right|_{k} \Omega M \Omega^{-1} \Omega=\left.\left.F\right|_{k} \Omega M \Omega^{-1}\right|_{k} \Omega=\left.F\right|_{k} \Omega=F^{*} .
$$

Formula (1.30) follows from (1.26). The remaining part of statement 1) is clear.

If $\varrho(D)=\left(\begin{array}{ll}A & B \\ C & D\end{array}\right)$, then, by $(1.27)$ and the definitions, we have

$$
\Omega \varrho(D)=\Omega \varrho(D) \Omega^{-1} \Omega=\varrho(A) \Omega=\varrho\left({ }^{t} D^{-1}\right) \Omega,
$$

because ${ }^{t} A D \equiv 1_{n}(\bmod m)$. The remaining part of 2$)$ follows from $(1.2)$

Identities (1.32)-(1.34) follow from (1.23), (1.31), and the definitions.

\section{§2. Twisting operators and Hecke operators}

Here we shall consider the interaction between the Hecke operators and the twisting operators $\mid \mathcal{T}(\chi, L)$, where $\chi$ is a Dirichlet character modulo $m$ and $L$ is a parameter matrix (2). First, we briefly recall the definitions of the Hecke-Shimura rings and the Hecke operators. For the details, see [4, Chapters 3 and 4].

Let $\Delta$ be a multiplicative semigroup and $\Lambda$ a subgroup of $\Delta$ such that every double coset $\Lambda M \Lambda$ of $\Delta$ modulo $\Lambda$ is a finite union of left cosets $\Lambda M^{\prime}$. Consider the vector space over a field (say, the field $\mathbb{C}$ of complex numbers) consisting of all formal finite linear combinations with coefficients in $\mathbb{C}$ of the symbols $(\Lambda M), M \in \Delta$, that are in one-to-one correspondence with the left cosets $\Lambda M$ of the set $\Delta$ modulo $\Lambda$. The group $\Lambda$ acts naturally on this space by the right multiplication defined on the symbols $(\Lambda M)$ by $(\Lambda M) \lambda=(\Lambda M \lambda)$ with $M \in \Delta$ and $\lambda \in \Lambda$. We denote by

$$
\mathcal{H}(\Lambda, \Delta)=\mathcal{H}_{\mathbb{C}}(\Lambda, \Delta)
$$

the subspace of all $\Lambda$-invariant elements. The multiplication of elements of $\mathcal{H}(\Lambda, \Delta)$, given by the formula

$$
\left(\sum_{\alpha} a_{\alpha}\left(\Lambda M_{\alpha}\right)\right)\left(\sum_{\beta} b_{\beta}\left(\Lambda M_{\beta}^{\prime}\right)\right)=\sum_{\alpha, \beta} a_{\alpha} b_{\beta}\left(\Lambda M_{\alpha} M_{\beta}^{\prime}\right),
$$


is independent of the choice of representatives $M_{\alpha}$ and $N_{\beta}$ in the corresponding left cosets and turns the linear space $\mathcal{H}(\Lambda, \Delta)$ into an associative algebra over $\mathbb{C}$ with the unity element $\left(\Lambda 1_{\Lambda}\right)$, called the Hecke-Shimura ring of $\Delta$ relative to $\Lambda$ (over $\mathbb{C}$ ). The elements

$$
T(M)=T(M)_{\Lambda}=(\Lambda M \Lambda)=\sum_{M^{\prime} \in \Lambda \backslash \Lambda M \Lambda}\left(\Lambda M^{\prime}\right) \quad(M \in \Delta),
$$

which are in one-to-one correspondence with the double cosets of $\Delta$ modulo $\Lambda$, belong to $\mathcal{H}(\Lambda, \Delta)$ and form a free basis of that ring over $\mathbb{C}$. For brevity, the symbols $(\Lambda M)$ and $T(M)$ will be referred to as the left and double classes (of $\Delta$ modulo $\Lambda$ ), respectively.

Now, suppose that the semigroup

$$
\Delta=\Delta^{n}=\mathbb{Z}_{2 n}^{2 n} \cap \mathbb{G}^{n}
$$

consists of all integral matrices contained in the group $\mathbb{G}^{n}$, and the group $\Lambda$ is a subgroup of finite index in the modular group $\Gamma^{n}$. Then the necessary conditions of the definition are satisfied, and we can define the Hecke-Shimura ring

$$
\mathcal{H}(\Lambda)=\mathcal{H}\left(\Lambda, \Delta^{n}\right)
$$

Next, we define a linear representation of this ring on the space $\mathfrak{M}_{k}(\Lambda)$ of modular forms of weight $k$ for the group $\Lambda$ by Hecke operators:

$$
\mathcal{H}(\Lambda) \ni T=\sum_{\alpha} a_{\alpha}\left(\Lambda M_{\alpha}\right): F \mapsto F|T=F|_{k} T=\left.\sum_{\alpha} a_{\alpha} F\right|_{k} M_{\alpha},
$$

where the $\left.\right|_{k} M_{\alpha}$ are the Petersson operators (1.1). The Hecke operators are independent of the choice of representatives in the corresponding left cosets and map the spaces $\mathfrak{M}_{k}(\Lambda)$ and $\mathfrak{N}_{k}(\Lambda)$ into themselves.

Largely, we shall be interested not in the entire Hecke-Shimura ring (2.3), but rather in certain subrings, called the $m$-regular subrings or simply the regular subrings, defined for the group $\Lambda$ of the form $\Gamma=\Gamma^{n}, \Gamma_{0}\left(m^{2}\right)=\Gamma_{0}^{n}\left(m^{2}\right)$, and $\widetilde{\Gamma}(m)=\widetilde{\Gamma}^{n}(m)$ as the Hecke-Shimura rings

$$
\mathcal{H}_{(m)}(\Lambda)=\mathcal{H}\left(\Lambda, \Delta_{(m)}(\Lambda)\right)
$$

of the group $\Lambda$ and the $m$-regular semigroups $\Delta_{(m)}(\Lambda)$ given, respectively, by the conditions

$$
\begin{gathered}
\Delta_{(m)}=\Delta_{(m)}(\Gamma)=\left\{M \in \Delta^{n} \mid \operatorname{gcd}(m, \mu(M))=1\right\} \\
\Delta_{(m)}\left(\Gamma_{0}\left(m^{2}\right)\right)=\left\{M=\left(\begin{array}{cc}
A & B \\
C & D
\end{array}\right) \in \Delta_{(m)}(\Gamma) \mid C \equiv \mathbf{0} \quad\left(\bmod m^{2}\right)\right\},
\end{gathered}
$$

and

$$
\Delta_{(m)}(\widetilde{\Gamma}(m))=\left\{M=\left(\begin{array}{cc}
A & B \\
C & D
\end{array}\right) \in \Delta_{(m)}\left(\Gamma_{0}\left(m^{2}\right)\right) \mid A \equiv \mathbf{1} \quad(\bmod m)\right\} .
$$

The corresponding Hecke operators are called the regular Hecke operators. It turns out that the rings (2.5) for these three groups are naturally isomorphic. More precisely, an easy modification of the proof of 4 , Theorem 3.3.3] yields the following statement.

Proposition 2.1. 1) The linear maps of the m-regular subrings $(2.6)-(2.8)$ defined on the double classes $(2.1)$ for each of the pairs $\left(\Lambda, \Lambda^{\prime}\right)$ of the form $\left(\widetilde{\Gamma}(m), \Gamma_{0}\left(m^{2}\right)\right),(\widetilde{\Gamma}(m), \Gamma)$, and $\left(\Gamma_{0}\left(m^{2}\right), \Gamma\right)$ by

$$
\mathcal{H}_{(m)}(\Lambda) \ni T_{\Lambda}(M) \mapsto T_{\Lambda^{\prime}}(M) \in \mathcal{H}_{(m)}\left(\Lambda^{\prime}\right) \quad\left(M \in \Delta_{(m)}(\Lambda)\right)
$$


are ring isomorphisms of the corresponding Hecke-Shimura rings. Moreover, the decompositions of the related double classes into left classes correspond naturally to each other:

$$
T_{\Lambda}(M)=\sum_{\alpha}\left(\Lambda M_{\alpha}\right) \Longrightarrow T_{\Lambda^{\prime}}(M)=\sum_{\alpha}\left(\Lambda^{\prime} M_{\alpha}\right) \quad\left(M \in \Delta_{(m)}(\Lambda)\right)
$$

2) The regular Hecke operators are compatible with the mappings (2.9) and the natural embeddings of the corresponding spaces of modular forms of weight $k$; namely, if $F \in \mathfrak{M}_{k}\left(\Lambda^{\prime}\right) \subset \mathfrak{M}_{k}(\Lambda)$ and $T_{\Lambda} \rightarrow T_{\Lambda^{\prime}}$, then

$$
F\left|T_{\Lambda}=F\right| T_{\Lambda^{\prime}} .
$$

Proposition 2.2. Each of the regular rings (2.5) for the groups $\Lambda$ of the form $\Gamma=$ $\Gamma^{n}, \Gamma_{0}\left(m^{2}\right)=\Gamma_{0}^{n}\left(m^{2}\right)$, or $\widetilde{\Gamma}(m)=\widetilde{\Gamma}^{n}(m)$ is a commutative integral domain generated over $\mathbb{C}$ by the algebraically independent elements

$$
\left\{\begin{array}{l}
T_{\Lambda}(p)=T_{\Lambda}(\operatorname{diag}(\underbrace{1, \ldots, 1}_{n}, \underbrace{p, \ldots, p}_{n})), \\
T_{\Lambda}^{j}\left(p^{2}\right)=T_{\Lambda}\left(\varrho_{j}(p) \operatorname{diag}(\underbrace{1, \ldots, 1}_{n-j}, \underbrace{p, \ldots, p}_{j}, \underbrace{p^{2}, \ldots, p^{2}}_{n-j}, \underbrace{p, \ldots, p}_{j})\right) \quad(1 \leq j \leq n),
\end{array}\right.
$$

where $p$ runs over all prime numbers not dividing $m$, and the elements

$$
\varrho_{j}(p)=\varrho_{j}^{n}(p)=\varrho(\operatorname{diag}(\underbrace{1, \ldots, 1}_{n-j}, \underbrace{p, \ldots, p}_{j})) \quad(1 \leq j \leq n)
$$

have the form (1.18).

Proof. For $\Lambda=\Gamma^{n}$ this claim was proved in [4, Theorem 3.3.23(1)]. The other cases follow from this by Proposition 2.1.

Theorem 2.3. For the groups $\Lambda=\widetilde{\Gamma}(m)$ and $\Lambda^{\prime}=\Gamma_{0}\left(m^{2}\right)$, let $M \in \Delta_{(m)}(\Lambda)$, and let $F \in \mathfrak{M}_{k}\left(\Lambda^{\prime}\right)$ be a modular form of weight $k$ for $\Lambda^{\prime}$. Let $\chi$ be a primitive Dirichlet character modulo $m$ and $L$ a matrix of the form (2). Then the following commutation relation holds true for the action of the Hecke operator $T_{\Lambda}(M)$ and the twist operator $\mathcal{T}(\chi, L)$ on the form $F$ :

$$
(F \mid \mathcal{T}(\chi, L))\left|T_{\Lambda}(M)=\chi(\mu(M))\left(F \mid T_{\Lambda^{\prime}}(M)\right)\right| \mathcal{T}(\chi, L) .
$$

Proof. By formulas (1.6), (2.1), (2.4), and (1.2), we obtain

$$
\begin{aligned}
(\mathcal{T}(\chi, L) F)\left|T_{\Lambda}(M)=\frac{g(\chi)}{m} \sum_{l \bmod m} \sum_{M^{\prime} \in \Lambda \backslash \Lambda M \Lambda} \bar{\chi}(-l) F\right|_{k} U\left(m^{-1} l L\right) M^{\prime} \\
=\frac{g(\chi)}{m} \sum_{l=0}^{m-1} \sum_{M^{\prime} \in \Lambda \backslash \Lambda M \Lambda} \chi(\mu(M)) \bar{\chi}(-l \mu(M)) \\
\quad \times\left.\left(\left.F\right|_{k} U\left(m^{-1} l L\right) M^{\prime} U\left(-m^{-1} l \mu(M) L\right)\right)\right|_{k} U\left(m^{-1} l \mu(M) L\right) \\
=\chi(\mu(M)) \frac{g(\chi)}{m} \sum_{l=0}^{m-1} \bar{\chi}\left(-l^{\prime}\right) \\
\left.\quad \times\left(\left.\sum_{M^{\prime} \in \Lambda \backslash \Lambda M \Lambda} F\right|_{k} U\left(m^{-1} l L\right) M^{\prime} U\left(-m^{-1} l^{\prime} L\right)\right)\right)\left.\right|_{k} U\left(m^{-1} l^{\prime} L\right),
\end{aligned}
$$

where $l^{\prime}=l \mu(M)$. By (1.13), for every matrix

$$
M^{\prime}=\left(\begin{array}{ll}
A^{\prime} & B^{\prime} \\
C^{\prime} & D^{\prime}
\end{array}\right) \in \Lambda M \Lambda \subset \Delta_{(m)}(\Lambda)
$$


and every $l=0,1, \ldots, m-1$, we have

(2.14)

$$
\begin{aligned}
M_{l}^{\prime} & =U\left(m^{-1} l L\right) M^{\prime} U\left(-m^{-1} l^{\prime} L\right) \\
& =\left(\begin{array}{cc}
A^{\prime}+m^{-1} l L C^{\prime} & -m^{-1}\left(l L D^{\prime}-l^{\prime} A^{\prime} L\right)+B^{\prime}-m^{-2} l l^{\prime} L C^{\prime} L \\
C^{\prime} & -m^{-1} l^{\prime} C^{\prime} L+D^{\prime}
\end{array}\right)=\left(\begin{array}{cc}
A_{l}^{\prime} & B_{l}^{\prime} \\
C_{l}^{\prime} & D_{l}^{\prime}
\end{array}\right) .
\end{aligned}
$$

Every such matrix belongs to $\Delta_{(m)}(\Lambda)$, because, clearly, the matrices $A_{l}^{\prime}, C_{l}^{\prime}$, and $D_{l}^{\prime}$ are integral matrices satisfying the congruences $A_{l}^{\prime} \equiv A^{\prime} \equiv 1_{n}(\bmod m)$ and $C_{l}^{\prime}=C^{\prime} \equiv 0_{n}$ $\left(\bmod m^{2}\right)$, and the matrix $B_{l}^{\prime}$ is integral, because $l L D^{\prime}-l^{\prime} A^{\prime} L \equiv l \mu(M) L-l^{\prime} L=$ $l^{\prime}(L-L) \equiv 0(\bmod m)$. By statement 2$)$ of Lemma 1.3 , we have

$$
U\left(m^{-1} l L\right) \Lambda U\left(-m^{-1} l L\right)=\Lambda \text { and } U\left(m^{-1} l^{\prime} L\right) \Lambda U\left(-m^{-1} l^{\prime} L\right)=\Lambda .
$$

Thus, the matrix $M_{l}^{\prime}$ ranges over the set

$$
U\left(m^{-1} l L\right)(\Lambda \backslash \Lambda M \Lambda) U\left(-m^{-1} l^{\prime} L\right)=\Lambda \backslash \Lambda\left(U\left(m^{-1} l L\right) M U\left(-m^{-1} l^{\prime} L\right)\right) \Lambda .
$$

It follows that for each $l$ we can write

$$
\left.\sum_{M^{\prime} \in \Lambda \backslash \Lambda M \Lambda} F\right|_{k} U\left(m^{-1} l L\right) M^{\prime} U\left(-m^{-1} l^{\prime} L\right)=F \mid T_{\Lambda}\left(U\left(m^{-1} l L\right) M U\left(-m^{-1} l^{\prime} L\right)\right) .
$$

By (2.11), the last-written image is equal to

$$
F \mid T_{\Lambda^{\prime}}\left(U\left(m^{-1} l L\right) M U\left(-m^{-1} l^{\prime} L\right)\right),
$$

and in order to complete the proof it suffices to show that, for every $l$, we have

$$
\Lambda^{\prime}\left(U\left(m^{-1} l L\right) M U\left(-m^{-1} l^{\prime} L\right)\right) \Lambda^{\prime}=\Lambda^{\prime} M \Lambda^{\prime}
$$

with $l^{\prime}=l \mu(M)$, because then each inner sum on the right in $(2.21)$ is equal to $F \mid T_{\Lambda^{\prime}}(M)$. As we have seen, the two matrices $M_{l}=U\left(m^{-1} l L\right) M U\left(-m^{-1} l^{\prime} L\right)$ and $M$ belong to the set $\Delta_{(m)}(\Lambda) \subset \Delta_{(m)}\left(\Lambda^{\prime}\right)$. Moreover, $\mu\left(M_{l}\right)=\mu(M)$. By [4, Lemma 3.3.6], (2.15) will be proved if we check that $M_{l}$ and $M$ have equal matrices of symplectic divisors, $\operatorname{sd}\left(M_{l}\right)=\operatorname{sd}(M)$. Put

$$
m U\left(m^{-1} l L\right)=\left(\begin{array}{cc}
m \cdot \mathbf{1} & l L \\
\mathbf{0} & m \cdot \mathbf{1}
\end{array}\right)=N, \quad m U\left(-m^{-1} l^{\prime} L\right)=\left(\begin{array}{cc}
m \cdot \mathbf{1} & -l^{\prime} L \\
\mathbf{0} & m \cdot \mathbf{1}
\end{array}\right)=N^{\prime} .
$$

Then

$$
U\left(m^{-1} l L\right)=\frac{1}{m} \gamma \operatorname{sd}(N) \gamma_{1}, \quad U\left(-m^{-1} l^{\prime} L\right)=\frac{1}{m} \gamma_{2} \operatorname{sd}\left(N^{\prime}\right) \gamma_{3},
$$

where $\gamma, \gamma_{1}, \gamma_{2}, \gamma_{3} \in \Gamma$. Hence, $M_{l}=\frac{1}{m^{2}} \gamma \operatorname{sd}(N) \gamma_{1} M \gamma_{2} \operatorname{sd}\left(N^{\prime}\right) \gamma_{3}$. It follows that

$$
m^{2} \operatorname{sd}(N)^{-1} \gamma^{-1} M_{l} \gamma_{3}^{-1}=\gamma_{1} M \gamma_{2} \operatorname{sd}\left(N^{\prime}\right) .
$$

Since $\mu(N)=\mu\left(N^{\prime}\right)=m^{2}$, the definition of symplectic divisors yields $\operatorname{sd}\left(m^{2} \operatorname{sd}(N)^{-1}\right)=$ $\operatorname{sd}(N), \operatorname{sd}\left(\gamma^{-1} M_{l} \gamma_{3}^{-1}\right)=\operatorname{sd}\left(M_{l}\right), \operatorname{sd}\left(\gamma_{1} M \gamma_{2}\right)=\operatorname{sd}(M)$, and $\operatorname{sd}\left(\operatorname{sd}\left(N^{\prime}\right)\right)=\operatorname{sd}\left(N^{\prime}\right)$. Since the number $\mu\left(M_{l}\right)=\mu(M)$ is coprime to $\mu(N)=\mu\left(N^{\prime}\right)=m^{2}$, relation (2.16) and the known properties of the matrices of symplectic divisors imply that

$$
\operatorname{sd}\left(m^{2} \operatorname{sd}(N)^{-1}\right) \operatorname{sd}\left(\gamma^{-1} M_{l} \gamma_{3}^{-1}\right)=\operatorname{sd}\left(\gamma_{1} M \gamma_{2}\right) \operatorname{sd}\left(\operatorname{sd}\left(N^{\prime}\right)\right)
$$

i.e.,

$$
\operatorname{sd}(N) \operatorname{sd}\left(M_{l}\right)=\operatorname{sd}(M) \operatorname{sd}\left(N^{\prime}\right)
$$

Obviously, this coincidence of diagonal matrices implies $\operatorname{sd}(N)=\operatorname{sd}\left(N^{\prime}\right)$ and $\operatorname{sd}\left(M_{l}\right)=$ $\operatorname{sd}(M)$, which proves (2.15) and the theorem. 
Corollary 2.4. In the notation of Theorem 2.3, if a modular form $F \in \mathfrak{M}_{k}\left(\Lambda^{\prime}\right)$ is an eigenfunction for the Hecke operator $\mid T_{\Lambda^{\prime}}(M), M \in \Delta_{(m)}(\Lambda)$, with the eigenvalue $\lambda(M)$, then the function $F \mid \mathcal{T}(\chi, L) \in \mathfrak{M}_{k}(\Lambda)$ is an eigenfunction for the operator $\mid T_{\Lambda}(M)$ with the eigenvalue $\chi(\mu(M)) \lambda(M)$.

Now we turn to relationships between regular Hecke operators and the star operator (1.29) defined with the help of a matrix $\Omega=\Omega^{n}(m)$ of the form (1.25) on the space $\mathfrak{M}_{k}(\widetilde{\Gamma}(m))$. For this, it will be convenient to split this space into invariant subspaces of the operators $\left.\right|_{k} \tau(d)=\left.\right|_{k} \tau^{n}(d)=\left.\right|_{k} \varrho(d \mathbf{1})$ with all $d$ prime to $m$ (see (1.24)). The mapping $\left.d \mapsto\right|_{k} \tau(d)$ determines a representation of the multiplicative Abelian group $G L_{1}(\mathbb{Z} / m \mathbb{Z})$ on the space $\mathfrak{M}_{k}(\widetilde{\Gamma}(m))$. Thus, this space is a direct sum of one-dimensional invariant subspaces. If $\left.F\right|_{k} \tau(d)=\psi(d) F$ with all $d$ prime to $m$, then $\psi$ is a character of $G L_{1}(\mathbb{Z} / m \mathbb{Z})$, which can be viewed as a Dirichlet character modulo $m$. Then we have the following direct sum decomposition:

$$
\mathfrak{M}_{k}(\widetilde{\Gamma}(m))=\bigoplus_{\psi \in \operatorname{Char}\left(G L_{1}(\mathbb{Z} / m \mathbb{Z})\right)} \mathfrak{M}_{k}(\widetilde{\Gamma}(m), \psi),
$$

where

$$
\mathfrak{M}_{k}(\widetilde{\Gamma}(m), \psi)=\left\{F \in \mathfrak{M}_{k}(\widetilde{\Gamma}(m))|F|_{k} \tau(d)=\psi(d) F, \operatorname{gcd}(d, m)=1\right\} .
$$

Note that, by Proposition 1.6, the image of the subspace $\mathfrak{M}\left(\Gamma_{0}\left(m^{2}\right)\right)$ under the twist operator $\mathcal{T}(\chi, L)$ with a character $\chi$ modulo $m$ and $p$-matrix $L$ is contained in the subspace (2.18) with $\psi=\chi^{2}$ :

$$
\mathfrak{M}_{k}\left(\Gamma_{0}\left(m^{2}\right)\right) \mid \mathcal{T}(\chi, L) \subset \mathfrak{M}_{k}\left(\widetilde{\Gamma}(m), \chi^{2}\right) .
$$

For regular Hecke operators on spaces of the form $\mathfrak{M}_{k}(\widetilde{\Gamma}(m), \psi)$, we now prove the following proposition.

Proposition 2.5. The following assertions are valid for the group $\Lambda=\widetilde{\Gamma}(m)$.

1) Suppose $F \in \mathfrak{M}_{k}(\Lambda, \psi)$ and $M \in \Delta_{(m)}(\Lambda)$. Then

$$
\left(F \mid T_{\Lambda}(M)\right)^{*}=\psi(\mu(M)) F^{*} \mid T_{\Lambda}\left(\tau(\mu(M)) M^{*}\right),
$$

where $\tau(\mu)=\tau^{n}(\mu), G^{*}=\left.G\right|_{k} \Omega$, and

$$
M^{*}=\Omega^{-1} M \Omega \quad\left(\Omega=\Omega^{n}(m)\right) .
$$

2) The matrix $\tau(\mu(M)) M^{*}$ belongs to $\Delta_{(m)}(\Lambda)$ together with $M$, and the mapping

$$
M \mapsto \check{M}=\tau(\mu(M)) M^{*}
$$

determines a bijection of the set $\Delta_{(m)}(\Lambda)$ that is identical on the sets of double cosets modulo $\Lambda$ contained in $\Delta_{(m)}(\Lambda)$, so that

$$
T_{\Lambda}(\check{M})=T_{\Lambda}(M) \quad \text { for all } \quad M \in \Delta_{(m)}(\Lambda) .
$$

In particular, relation (2.20) can be rewritten in the form

$$
\left(F \mid T_{\Lambda}(M)\right)^{*}=\psi(\mu(M)) F^{*} \mid T_{\Lambda}(M) \quad\left(M \in \Delta_{(m)}(\Lambda)\right) .
$$


Proof. Using (1.29), (2.1), (2.4), (1.31), and (1.2), we obtain

$$
\begin{aligned}
\left(F \mid T_{\Lambda}(M)\right)^{*} & =F\left|T_{\Lambda}(M)\right| \Omega=\left.\sum_{M^{\prime} \in \Lambda \backslash \Lambda M \Lambda} F\right|_{k} M^{\prime} \Omega \\
& =\left.\sum_{M^{\prime} \in \Lambda \backslash \Lambda M \Lambda} F\right|_{k} \Omega \tau(\mu(M))^{-1} \Omega^{-1} \Omega \tau(\mu(M)) \Omega^{-1} M^{\prime} \Omega \\
& =\left.\sum_{M^{\prime} \in \Lambda \backslash \Lambda M \Lambda} F\right|_{k} \tau(\mu(M)) \Omega \tau(\mu(M))\left(M^{\prime}\right)^{*} \\
& =\left.\psi(\mu(M)) \sum_{M^{\prime} \in \Lambda \backslash \Lambda M \Lambda} F^{*}\right|_{k} \tau(\mu(M))\left(M^{\prime}\right)^{*} \\
& =\left.\psi(\mu(M)) \sum_{M^{\prime \prime} \in \Lambda \backslash \Lambda \tau(\mu(M)) M^{*} \Lambda} F^{*}\right|_{k} M^{\prime \prime}=\psi(\mu(M)) F^{*} \mid T_{\Lambda}\left(\tau(M) M^{*}\right),
\end{aligned}
$$

because $\Omega^{-1} \Lambda \Omega=\Lambda$, which proves part 1$)$.

The fact that $\check{M} \in \Delta_{(m)}(\Lambda)$ follows from the definitions. By (1.26) and (1.31), we have $\left(M^{*}\right)^{*}=M$ and

$$
\check{M}=M \quad \text { for all } M \in \Delta_{(m)}(\Lambda) .
$$

Thus, the mapping (2.22) is a bijection. By Proposition 2.1 for the pair $(\widetilde{\Gamma}(m), \Gamma)$, for the proof of (2.23) it suffices to check that $\check{M} \in \Gamma M \Gamma$, or that the matrices $\check{M}$ and $M$ have equal matrices of symplectic divisors, $\operatorname{sd}(\check{M})=\operatorname{sd}(M)$ (see [4, Lemma 3.3.6]). The definition shows that $\check{M} m \Omega^{-1}=\tau(\mu(M)) m \Omega^{-1} M$. Since the multiplier $\mu(M)=\mu(\check{M})$ is coprime to the multiplier $\mu\left(m \Omega^{-1}\right)=m^{2}$, we can use the well-known properties of symplectic divisors to obtain

$$
\operatorname{sd}\left(\check{M} m \Omega^{-1}\right)=\operatorname{sd}(\check{M}) \operatorname{sd}\left(m \Omega^{-1}\right)=\operatorname{sd}\left(\tau(\mu(M)) m \Omega^{-1} M\right)=\operatorname{sd}\left(m \Omega^{-1}\right) \operatorname{sd}(M) .
$$

Hence, $\operatorname{sd}(\check{M})=\operatorname{sd}(M)$.

Note that relation (1.31) implies

$$
F^{*} \in \mathfrak{M}_{k}(\widetilde{\Gamma}(m), \bar{\psi}) \quad \text { if } F \in \mathfrak{M}_{k}(\widetilde{\Gamma}(m), \psi),
$$

where $\bar{\psi}$ denotes the character conjugate to $\psi$.

Corollary 2.6. A modular form $F \in \mathfrak{M}_{k}(\widetilde{\Gamma}(m), \psi)$ is an eigenfunction for the Hecke operator $\mid T_{\Lambda}(M)$, where $\Lambda=\widetilde{\Gamma}(m)$ and $M \in \Delta_{(m)}(\Lambda)$, with the eigenvalue $\lambda_{F}(M)$ if and only if the function $F^{*} \in \mathfrak{M}(\widetilde{\Gamma}(m), \bar{\psi})$ is an eigenfunction for the operator $\mid T_{\Lambda}(M)$ with the eigenvalue

$$
\lambda_{F^{*}}(M)=\bar{\psi}(\mu(M)) \lambda_{F}(M) .
$$

Proof. Since $\lambda_{F}(M) F=F \mid T_{\Lambda}(M),(2.24)$ yields

$$
\lambda_{F}(M) F^{*}=\left(\lambda_{F}(M) F\right)^{*}=\left(F \mid T_{\Lambda}(M)\right)^{*}=\psi(\mu(M)) F^{*} \mid T_{\Lambda}(M),
$$

which proves (2.27).

\section{§3. REgular ZETA-FUnCtions of TWISTED FORMS AND $L$-FUnCTIONS}

We consider sums of all different double cosets of fixed multipliers prime to $m$ of $m$-regular Hecke-Shimura rings $\mathcal{H}_{(m)}(\Lambda)$ of the form (2.5) for the groups $\Lambda$ defined by conditions (1.8), (1.10), or (1.11):

$$
T_{\Lambda}(a)=\sum_{M \in \Lambda \backslash \Delta_{(m)}(\Lambda) / \Lambda, \mu(M)=a} T_{\Lambda}(M) \quad \text { with } a \text { prime to } m .
$$


Theorem 3.1. The elements (3.1) for the groups $\Lambda$ of the form $\Gamma, \Gamma_{0}\left(m^{2}\right)$, or $\widetilde{\Gamma}(m)$ satisfy the following rules:

$$
T_{\Lambda}(a) T_{\Lambda}\left(a^{\prime}\right)=T_{\Lambda}\left(a a^{\prime}\right) \quad \text { if } \operatorname{gcd}\left(a, a^{\prime}\right)=1 ;
$$

for each prime number $p$ not dividing $m$, the formal power series over the ring $\mathcal{H}_{(m)}(\Lambda)$ with the coefficients $T_{\Lambda}(1), T_{\Lambda}(p), T_{\Lambda}\left(p^{2}\right), \ldots$ is formally equal to a rational fraction with coefficients in $\mathcal{H}_{(m)}(\Lambda)$ and with denominator and numerator of degree $2^{n}$ and at most $2^{n}-2$, respectively,

$$
\sum_{\delta=0}^{\infty} T_{\Lambda}\left(p^{\delta}\right) t^{\delta}=Q_{p, \Lambda}(t)^{-1} R_{p, \Lambda}(t)
$$

where

$$
Q_{p, \Lambda}(t)=\sum_{i=0}^{2^{n}}(-1)^{i} \mathbf{q}_{\Lambda}^{i}(p) t^{i}, \quad R_{p, \Lambda}(t)=\sum_{i=0}^{2^{n}-2}(-1)^{i} \mathbf{r}_{\Lambda}^{i}(p) t^{i},
$$

and the coefficients $\mathbf{q}_{\Lambda}^{i}(p)$ and $\mathbf{r}_{\Lambda}^{i}(p)$ belong to $\mathcal{H}_{(m)}(\Lambda)$. Moreover, the coefficients of the denominator $Q_{p, \Lambda}(t)$ satisfy the relations

$$
\mathbf{q}_{\Lambda}^{0}(p)=[\mathbf{1}]_{\Lambda}, \quad \mathbf{q}_{\Lambda}^{1}(p)=T_{\Lambda}(p), \quad \mathbf{q}_{\Lambda}^{2^{n}}(p)=\left(p^{n(n+1) / 2}[\mathbf{p}]_{\Lambda}\right)^{2^{n-1}}
$$

and the symmetry relations

$$
\mathbf{q}_{\Lambda}^{2^{n}-i}(p)=\left(p^{n(n+1) / 2}[\mathbf{p}]_{\Lambda}\right)^{2^{n-1}-i} \mathbf{q}_{\Lambda}^{i}(p) \quad\left(0 \leq i \leq 2^{n}\right),
$$

where

$$
[\mathbf{a}]_{\Lambda}=T_{\Lambda}(a \tau(a))
$$

and $\tau(a)=\tau^{n}(a)$ has the form (1.24).

Proof. In accordance with the isomorphism of the rings $\mathcal{H}_{(m)}(\Lambda)$ and $\mathcal{H}_{(m)}(\Gamma)$ described in Proposition 2.1, it suffices to prove the theorem for the group $\Lambda=\Gamma=\Gamma^{n}$ only. But for the group $\Gamma^{n}$ all the claims are well known: an analog of (3.2) was proved in [7, an analog of the summation formula (3.3) was established in [1], and relations similar to (3.5)-(3.6) were checked in [4, §3.3.3].

From (3.2) and (3.3) it follows that the formal Dirichlet series with the coefficients $\eta(a) T_{\Lambda}(a)$ for $a$ prime to $m$, where $\eta(a)$ is a completely multiplicative complex-valued function of $a$, can be expanded in a formal Euler product:

$$
\begin{aligned}
D_{\Lambda}(s, \eta) & =\sum_{a \in \mathbb{N}, \operatorname{gcd}(a, m)=1} \frac{\eta(a) T_{\Lambda}(a)}{a^{s}} \\
& =\prod_{\text {primes } p \nmid m} \sum_{\delta=0}^{\infty} \eta(p)^{\delta} T_{\Lambda}\left(p^{\delta}\right) p^{-\delta s}=\prod_{\text {primes } p \nmid m} Q_{p, \Lambda}\left(\eta(p) p^{-s}\right)^{-1} R_{p, \Lambda}\left(\eta(p) p^{-s}\right) \\
& =\left(\prod_{\text {primes } p \nmid m} Q_{p, \Lambda}\left(\eta(p) p^{-s}\right)\right)^{-1}\left(\prod_{\text {primes } p \nmid m} R_{p, \Lambda}\left(\eta(p) p^{-s}\right)\right)
\end{aligned}
$$

where $a^{s}$ is regarded as a formal quasicharacter of the multiplicative semigroup $\mathbb{N}$ of positive integers. (We recall that, by Proposition 2.2, the $\operatorname{ring} \mathcal{H}_{(m)}(\Lambda)$ is commutative.) 
Now we turn to regular Hecke operators on the spaces $\mathfrak{M}_{k}(\Lambda)$ of modular forms of weight $k$ for these groups $\Lambda$. Suppose we are given an eigenfunction $F \in \mathfrak{M}_{k}(\Lambda)$ for all Hecke operators $\mid T$ with $T \in \mathcal{H}_{(m)}(\Lambda)$,

$$
F \mid T=\lambda_{F}(T) F \quad\left(T \in \mathcal{H}_{(m)}(\Lambda)\right) .
$$

We set $\lambda_{F}\left(T_{\Lambda}(a)\right)=\lambda_{F}(a)$, so that

$$
F \mid T_{\Lambda}(a)=\lambda_{F}(a) F \quad(\operatorname{gcd}(a, m)=1) .
$$

Replacing all coefficients $T \in \mathcal{H}_{(m)}(\Lambda)$ in the formal identity (3.8) with the eigenvalues $\lambda_{F}(T)$ of the corresponding Hecke operators acting on the eigenfunction $F$, we obtain a formal Euler product expansion over $\mathbb{C}$ of the form

$$
\begin{aligned}
D_{F}(s, \eta) & =\sum_{a \in \mathbb{N}, \operatorname{gcd}(a, m)=1} \frac{\eta(a) \lambda_{F}(a)}{a^{s}} \\
& =\left(\prod_{\text {primes } p \nmid m} Q_{p, F}\left(\eta(p) p^{-s}\right)\right)^{-1}\left(\prod_{\text {primes } p \nmid m} R_{p, F}\left(\eta(p) p^{-s}\right)\right),
\end{aligned}
$$

where

$$
Q_{p, F}(t)=\sum_{i=0}^{2^{n}}(-1)^{i} \lambda_{F}\left(\mathbf{q}_{\Lambda}^{i}(p)\right) t^{i}, \quad R_{p, F}(t)=\sum_{i=0}^{2^{n}-2}(-1)^{i} \lambda_{F}\left(\mathbf{r}_{\Lambda}^{i}(p)\right) t^{i} .
$$

Assuming now that the function $\eta(a)$ grows not faster than a constant power of $a$, and using the known estimates of Fourier coefficients of modular forms, it is not hard to show that the infinite series and products occurring in the formal identity (3.11) converge absolutely and uniformly in a right half-plane $\operatorname{Re} s>c=c(F, \eta)$ of the complex variable $s$, thus yielding holomorphic functions in $s$ there. (For the case of the group $\Lambda=\Gamma^{n}$, see [3. §1.3].) We shall call the function

$$
L_{F}(s, \eta)=\left(\prod_{\text {primes } p \nmid m} Q_{p, F}\left(\eta(p) p^{-s}\right)\right)^{-1}
$$

the (regular) L-function of the eigenfunction $F$ with "character" $\eta$ and call the function

$$
Z_{F}(s)=L_{F}(s, 1)=\left(\prod_{\text {primes } p \nmid m} Q_{p, F}\left(p^{-s}\right)\right)^{-1}
$$

the (regular) zeta-function of the eigenfunction $F$. Proposition 2.1 shows that the Euler products (3.13) and (3.14) do not depend on the choice of a group $\Lambda$ such that the space $\mathfrak{M}_{k}(\Lambda)$ contains the eigenfunction $F$.

Theorem 3.2. For the group $\Lambda^{\prime}=\Gamma_{0}\left(m^{2}\right)=\Gamma_{0}^{n}\left(m^{2}\right)$, let $F \in \mathfrak{M}_{k}\left(\Lambda^{\prime}\right)$ be an eigenfunction for all Hecke operators $\mid T_{\Lambda^{\prime}}(M)$ with $M \in \Delta_{(m)}\left(\Lambda^{\prime}\right)$. Then, for every primitive Dirichlet character $\chi$ modulo $m$ and every p-matrix $L$, the twisted form $F \mid \mathcal{T}(\chi, L) \in$ $\mathfrak{M}_{k}(\Lambda)$, where $\Lambda=\widetilde{\Gamma}(m)$, is an eigenfunction for all Hecke operators $\mid T_{\Lambda}(M)$ with $M \in \Delta_{(m)}(\Lambda)$, and the zeta-function of the twisted form in every domain of absolute convergence is equal to the $L$-function of the form $F$ with character $\chi$ :

$$
Z_{F \mid T(\chi, L)}(s)=L_{F}(s, \chi) .
$$

Moreover, the form $(F \mid \mathcal{T}(\chi, L))^{*}$, where the star denotes the mapping (1.29), is also an eigenfunction for all Hecke operators $\mid T_{\Lambda}(M)$ with $M \in \Delta_{(m)}(\Lambda)$, and the zeta-function 
of this form in every domain of absolute convergence is equal to the L-function of the form $F$ with the conjugate character $\bar{\chi}$ :

$$
Z_{(F \mid T(\chi, L))^{*}}(s)=L_{F}(s, \bar{\chi}) .
$$

Proof. By Corollary 2.4, the relation $F \mid T_{\Lambda^{\prime}}(a)=\lambda_{F}(a) F$ implies $(F \mid \mathcal{T}(\chi, L)) T_{\Lambda}(a)=$ $\chi(a) \lambda_{F}(a) F$ for each $a$ prime to $m$. Hence, by (3.11) and (3.14), for each prime $p$ not dividing $m$ we obtain

$$
Q_{p, F \mid T(\chi, L)}(t)=Q_{p, F}(\chi(p) t)
$$

and

$$
\begin{aligned}
Z_{F \mid T(\chi, L)}(s) & =\left(\prod_{\text {primes } p \nmid m} Q_{p, F \mid T(\chi, L)}\left(p^{-s}\right)\right)^{-1} \\
& =\left(\prod_{\text {primes } p \nmid m} Q_{p, F}\left(\chi(p) p^{-s}\right)\right)^{-1}=L_{F}(s, \chi) .
\end{aligned}
$$

In order to prove (3.16), we note that, by (2.19) and Corollary 2.6, for each $a$ prime to $m$ we get

$$
\lambda_{(F \mid T(\chi, L))^{*}}(a)=\overline{\chi^{2}(a)} \lambda_{F \mid T(\chi, L)}(a) .
$$

Therefore, by (3.17), for each prime $p$ not dividing $m$ we have

$$
\left.Q_{p,(F \mid T(\chi, L))^{*}}(t)=Q_{p, F \mid T(\chi, L)}\left(\overline{\chi^{2}}(p) t\right)=Q_{p, F}\left(\chi(p) \overline{\chi^{2}}(p)\right) t\right)=Q_{p, F}(\bar{\chi}(p) t),
$$

and (3.16) is true.

Note that the definition (2.18) of the subspaces $\mathfrak{M}_{k}(\widetilde{\Gamma}(m), \psi)$ implies easily that every modular form $F \in \mathfrak{M}_{k}(\widetilde{\Gamma}(m), \psi)$ is an eigenfunction of each Hecke operator $\mid[\mathbf{a}]_{\Lambda}$ corresponding to the element (3.7) for $\Lambda=\widetilde{\Gamma}(m)$ with the eigenvalue

$$
\begin{gathered}
\psi(a) a^{2(n k-\langle n\rangle)} \operatorname{det}(a \cdot \mathbf{1})^{-k}=\psi(a) a^{n k-n(n+1)}, \\
F \mid[\mathbf{a}]_{\Lambda}=\psi(a) a^{n k-n(n+1)} F \quad\left(F \in \mathfrak{M}_{k}(\widetilde{\Gamma}(m), \psi) \text { and } a \text { prime to } m\right) ;
\end{gathered}
$$

see (6). By (2.19), the twist with character $\chi$ of every modular form of this space or the space $\mathfrak{M}_{k}\left(\Gamma_{0}\left(m^{2}\right)\right)$ belongs to $\mathfrak{M}_{k}\left(\widetilde{\Gamma}(m), \chi^{2}\right)$. Hence, for the twisted forms we obtain

$$
(F \mid \mathcal{T}(\chi, L)) \mid[\mathbf{a}]_{\Lambda}=\chi\left(a^{2}\right) a^{n k-n(n+1)}(F \mid \mathcal{T}(\chi, L)) \text { for all } a \text { prime to } m .
$$

These relations together with (3.5), (3.6) allow us to compute the constant and the leading coefficients in the denominators of $p$-factors of zeta-functions for the twisted form $G=F \mid \mathcal{T}(\chi, L)$ of an eigenfunction $F \in \mathfrak{M}_{k}\left(\Gamma_{0}\left(m^{2}\right)\right)$ :

$$
\lambda_{G}\left(\mathbf{q}_{\Lambda}^{0}(p)\right)=1, \quad \lambda_{G}\left(\mathbf{q}_{\Lambda}^{2^{n}}(p)\right)=\left(p^{n k-\langle n\rangle} \chi\left(p^{2}\right)\right)^{2^{n-1}}
$$

and to write the general symmetry relations for the coefficients of the denominators:

$$
\lambda_{G}\left(\mathbf{q}_{\Lambda}^{2^{n}-i}(p)\right)=\left(p^{n k-\langle n\rangle} \chi\left(p^{2}\right)\right)^{2^{n-1}-i} \lambda_{G}\left(\mathbf{q}_{\Lambda}^{i}(p)\right) \quad\left(0 \leq i \leq 2^{n}\right) .
$$




\section{$\S 4$. L-FUNCTIONS OF CUSP FORMS OF GENUS ONE}

Here we apply Theorem 3.2 on reduction to the simplest case of modular forms in one variable and reprove some consequences of the Atkin-Lehner theory of new forms [5]. In this section we assume that the genus $n$ is equal to 1 .

Let $F \in \mathfrak{N}_{k}\left(\Gamma_{0}\left(m^{2}\right)\right)$ be a cusp form of an integral weight $k$ for the congruence subgroup of the modular group $\Gamma^{1}$ of the form $\Gamma_{0}\left(m^{2}\right)=\Gamma_{0}^{1}\left(m^{2}\right)$ with Fourier expansion (1) for $n=1$,

$$
F(z)=\sum_{a=1}^{\infty} f(a) e^{2 \pi i a z} \quad\left(z=x+i y \in \mathbb{H}=\mathbb{H}^{1}\right) .
$$

We assume that $F$ is an eigenfunction for all Hecke operators $|T=| T_{\Lambda^{\prime}}$ with $T \in$ $\mathcal{H}_{(m)}\left(\Lambda^{\prime}\right)$, where $\Lambda^{\prime}=\Gamma_{0}\left(m^{2}\right)$ :

$$
F \mid T=\lambda_{F}(T) F \quad\left(T=T_{\Lambda^{\prime}} \in \mathcal{H}_{(m)}\left(\Lambda^{\prime}\right)\right) .
$$

Next, we denote by $G$ the image of $F$ under the twist operator (4) with a fixed primitive Dirichlet character $\chi$ modulo $m$ and the $p$-matrix $L=l=1$; by definition, $G$ has the Fourier expansion

$$
G(z)=\sum_{a=1}^{\infty} g(a) e^{2 \pi i a z}, \quad \text { where } g(a)=\chi(a) f(a),
$$

and, by Proposition 1.4, it belongs to the space $\mathfrak{N}_{k}(\widetilde{\Gamma}(m))$ of cusp forms of weight $k$ for the group $\widetilde{\Gamma}(m)=\widetilde{\Gamma}^{1}(m)$ of the form (1.11),

$$
G=F|\mathcal{T}(\chi)=F| \mathcal{T}(\chi, 1) \in \mathfrak{N}_{k}(\widetilde{\Gamma}(m))
$$

Then, by Corollary 2.4, the form $G$ is an eigenfunction for all Hecke operators $\mid T_{\Lambda}$ with $\Lambda=\widetilde{\Gamma}(m)$ and $T_{\Lambda} \in \mathcal{H}_{(m)}(\Lambda)$. Moreover, the eigenvalues of the Hecke operators corresponding to the elements (3.1) for $\Lambda=\widetilde{\Gamma}(m)$ and $\Lambda^{\prime}=\Gamma_{0}\left(m^{2}\right)$ acting on $G$ and $F$, respectively, satisfy

$$
\lambda_{G}(a)=\chi(a) \lambda_{F}(a) \quad(a \in \mathbb{N}, \operatorname{gcd}(a, m)=1) .
$$

Thus, we have two sequences of complex numbers associated with each of the eigenfunctions: the sequence of Fourier coefficients, and the sequence of eigenvalues of Hecke operators. The natural question is whether these sequences are related to each other. The question is interesting in two respects: first, because of multiplicative properties of Hecke operators and their eigenvalues, such relations could reveal multiplicative properties of Fourier coefficients, which often presents an arithmetical interest, and second, analytic properties of modular forms as the generating series for their Fourier coefficients may possibly be transferred to analytic properties of generating functions for the eigenvalues and corresponding Euler products.

The relationship between Fourier coefficients of an eigenfunction and the corresponding eigenvalues, for modular forms in one variable, were discovered by Hecke and are quite simple.

Lemma 4.1 (Hecke). Suppose that a modular form $F \in \mathfrak{N}_{k}\left(\Gamma_{0}\left(m^{2}\right)\right)$ with Fourier expansion (4.1) is an eigenfunction of the Hecke operator $T(a)$ for the group $\Gamma_{0}\left(m^{2}\right)$ with a prime to $m$, and let $\lambda_{F}(a)$ be the corresponding eigenvalue. Then, for every $b=1,2, \ldots$, we have

$$
\lambda_{F}(a) f(b)=\sum_{d \mid a, b} d^{k-1} f\left(\frac{a b}{d^{2}}\right),
$$


where $d$ ranges over all positive common divisors of a and $b$; in particular,

$$
f(a)=f(1) \lambda_{F}(a) .
$$

Proof. The proof for an arbitrary $m$ is actually the same as that in the case where $m=1$; see, e.g., 6, Chapter II, Proposition 7].

From this lemma and the well-known estimates of Fourier coefficients of cusp forms we obtain the following Euler product expansions for the generating Dirichlet series of Fourier coefficients of eigenforms.

Proposition 4.2. Suppose that a cusp form $F \in \mathfrak{N}_{k}\left(\Gamma_{0}\left(m^{2}\right)\right)$ with Fourier expansion (4.1) is an eigenfunction of all regular Hecke operators of the form $T(a)$ for the group $\Gamma_{0}\left(m^{2}\right)$ with the eigenvalues $\lambda_{F}(a)$, and $\chi$ is a primitive Dirichlet character modulo $m \geq 1$. Then the following identity is valid in the right half-plane $\operatorname{Re} s>k / 2+1$ :

$$
\begin{aligned}
\sum_{a=1}^{\infty} \frac{\chi(a) f(a)}{a^{s}} & =f(1) \prod_{\text {primes } p \nmid m}\left(1-\chi(p) \lambda_{F}(p) p^{-s}+\chi^{2}(p) p^{k-1-2 s}\right)^{-1} \\
& =f(1) L_{F}(s, \chi),
\end{aligned}
$$

where $L_{F}(s, \chi)$ is the L-function (3.13) of the form $F$.

Proof. Using (4.7), we get

$$
\sum_{a=1}^{\infty} \frac{\chi(a) f(a)}{a^{s}}=f(1) \sum_{a=1}^{\infty} \frac{\chi(a) \lambda_{F}(a)}{a^{s}}=f(1) L_{F}(s, \chi)
$$

(note that $\chi(a)=0$ if $a$ is not prime to $m$ ).

Identity (4.8) makes it possible to investigate the analytic properties of the $L$-functions $L_{F}(s, \chi)$. By Theorem 3.2, it suffices to consider the zeta-function of the twisted form $G$.

Proposition 4.3. Suppose that a cusp form $F$ of weight $k$ for the group $\Gamma_{0}\left(m^{2}\right)$ is an eigenfunction of all regular Hecke operators for that group. Let $G=F \mid \mathcal{T}(\chi) \in \mathfrak{M}_{k}(\widetilde{\Gamma}(m))$ be the twist (4.3) of $F$ with a primitive Dirichlet character $\chi$ modulo $m \geq 1$. Then the following is true.

1) The zeta-function (3.14) of the eigenfunction $G$ in the half-plane $\operatorname{Re} s>k / 2+1$ satisfies the identity

$$
\begin{aligned}
\Psi_{G}(s) & =m^{s-k / 2}(2 \pi)^{-s} \Gamma(s) Z_{G}(s) \\
& =\frac{1}{g(1)}\left(m^{s-k / 2} \int_{1 / m}^{\infty} y^{s-1} G(i y) d y+(-i)^{k} m^{k / 2-s} \int_{1 / m}^{\infty} y^{k-1-s} G^{*}(i y) d y\right),
\end{aligned}
$$

where $g(1)$ is the first coefficient of the Fourier expansion (4.4) of $G, \Gamma(s)$ is the gammafunction, and $G \mapsto G^{*}$ is the mapping (1.29).

2) The right-hand side of (4.9) is holomorphic for all s. Thus, the function $\Psi_{G}(s)$ has analytic continuation to the entire s-plane as a holomorphic function.

3) The function $\Psi_{G}(s)$ satisfies the functional equation

$$
\Psi_{G}(k-s)=(-i)^{k} \frac{g^{*}(1)}{g(1)} \Psi_{G^{*}}(s),
$$

where, for the eigenfunction $G^{*}=(F \mid \mathcal{T}(\chi))^{*} \in \mathfrak{N}_{k}(\widetilde{\Gamma}(m))$ of all regular Hecke operators, 
we set

$$
\Psi_{G^{*}}(s)=m^{s-k / 2}(2 \pi)^{-s} \Gamma(s) Z_{G^{*}}(s),
$$

$Z_{G^{*}}(s)$ is the zeta-function of $G^{*}$, and $g^{*}(1)$ is the first Fourier coefficient of $G^{*}$.

Proof. By Theorem 3.2 and formulas (4.3), we can write identity (4.8) in the form

$$
R_{G}(s)=\sum_{a=1}^{\infty} \frac{g(a)}{a^{s}}=\sum_{a=1}^{\infty} \frac{\chi(a) f(a)}{a^{s}}=f(1) L_{F}(s, \chi)=f(1) Z_{G}(s) .
$$

Using the Euler integral

$$
\int_{0}^{\infty} y^{s-1} e^{-\alpha y} d y=\Gamma(s) \alpha^{-s} \quad(\alpha>0, \operatorname{Re} s>0)
$$

where $\Gamma(s)$ is the gamma-function, we obtain

$$
\begin{aligned}
\int_{0}^{\infty} y^{s-1} G(i y) d y & =\sum_{a=1}^{\infty} g(a) \int_{0}^{\infty} y^{s-1} e^{-2 \pi a y} d y \\
& =(2 \pi)^{-s} \Gamma(s) \sum_{a=1}^{\infty} \frac{g(a)}{a^{s}}=(2 \pi)^{-s} \Gamma(s) R_{G}(s) \quad(\operatorname{Re} s>k / 2+1) .
\end{aligned}
$$

Hence, by (4.12),

$$
\begin{aligned}
\Psi_{G}(s) & =\frac{1}{g(1)} m^{s-k / 2}(2 \pi)^{-s} \Gamma(s) R_{G}(s)=\frac{1}{g(1)} m^{s-k / 2} \int_{0}^{\infty} y^{s-1} G(i y) d y \\
& =\frac{1}{g(1)}\left(m^{s-k / 2} \int_{1 / m}^{\infty} y^{s-1} G(i y) d y+m^{s-k / 2} \int_{0}^{1 / m} y^{s-1} G(i y) d y\right) .
\end{aligned}
$$

Replacing $y$ by $1 / m^{2} y$, we can write

$$
\begin{aligned}
\int_{0}^{1 / m} y^{s-1} G(i y) d y & =\int_{\infty}^{1 / m}\left(m^{2} y\right)^{1-s} G\left(i / m^{2} y\right) \frac{-d y}{m^{2} y^{2}} \\
& =(-i)^{k} m^{k-2 s} \int_{1 / m}^{\infty} y^{k-1-s}(-i m y)^{-k} G\left(i / m^{2} y\right) d y \\
& =(-i)^{k} m^{k-2 s} \int_{1 / m}^{\infty} y^{k-1-s} G^{*}(i y) d y,
\end{aligned}
$$

because, by definition,

$$
G^{*}(z)=\left.G\right|_{k}\left(\begin{array}{cc}
0 & 1 / m \\
m & 0
\end{array}\right)=(-m z)^{-k} G\left(-1 / m^{2} z\right)
$$

Substituting this in the right-hand side of (4.13), we arrive at (4.9).

Since $G$ is a cusp form, the absolute values of the two integrands on the right in (4.9) decay exponentially as $y \rightarrow+\infty$. Hence, both integrals converge absolutely and uniformly for all $s \in \mathbb{C}$ and determine functions holomorphic everywhere. 
Finally, by formula (4.9) with $G^{*}$ in place of $G$ and $k-s$ in place of $s$, we get

$$
\begin{aligned}
\Psi_{G^{*}}(k-s)= & \frac{1}{g^{*}(1)}\left(m^{k-s-k / 2} \int_{1 / m}^{\infty} y^{k-s-1} G^{*}(i y) d y\right. \\
& \left.+(-i)^{k} m^{k / 2-(k-s)} \int_{1 / m}^{\infty} y^{k-1-(k-s)} G^{* *}(i y) d y\right) \\
& =i^{k} \frac{g(1)}{g^{*}(1)} \frac{1}{g(1)}\left((-i)^{k} \int_{1 / m}^{\infty} y^{k-s-1} G^{*}(i y) d y+m^{s-k / 2} \int_{1 / m}^{\infty} y^{s-1} G(i y) d y\right) \\
& =i^{k} \frac{g(1)}{g^{*}(1)} \Psi_{G}(s),
\end{aligned}
$$

because, by (1.26),

$$
G^{* *}=\left.\left.G\right|_{k} \Omega\right|_{k} \Omega=\left.\left.G\right|_{k} \Omega^{2} G\right|_{k}\left(-1_{2}\right)=(-1)^{k} G .
$$

The functional equation (4.10) follows.

Returning to $L$-functions, now we can prove the following theorem.

Theorem 4.4. In the notation and under the assumptions of Proposition 4.2, the function

$$
\Psi_{F}(s, \chi)=m^{s-k / 2}(2 \pi)^{-s} \Gamma(s) L_{F}(s, \chi)
$$

has analytic continuation to the entire s-plane as a holomorphic function and satisfies the functional equation

$$
\Psi_{F}(k-s, \chi)=(-i)^{k} \frac{g^{*}(1)}{f(1)} \Psi_{F}(s, \bar{\chi}),
$$

where $f(1)$ and $g^{*}(1)$ are the first Fourier coefficients of $F$ and of $(F \mid \mathcal{T}(\chi))^{*}$, respectively.

Proof. By Theorem 3.2, in the notation of Proposition 4.3 we have $\Psi_{F}(s, \chi)=\Psi_{G}(s)$, and the functional equation (4.15) follows from (4.10), because

$$
g(1)=\chi(1) f(1)=f(1)
$$

by (4.3), and

$$
Z_{G^{*}}(s)=L(s, \bar{\chi})
$$

by $(3.16)$.

\section{REFERENCES}

[1] A. N. Andrianov, Spherical functions for $G L_{n}$ over local fields, and the summation of Hecke series, Mat. Sb. (N. S.) 83(125) (1970), no. 3, 429-451; English transl., Math. USSR-Sb. 12 (1970), 429452. MR0282982(44:216)

[2] - Dirichlet series with Euler product in the theory of Siegel modular forms of genus two, Trudy Mat. Inst. Steklov. 112 (1971), 73-94; English transl. in Proc. Steklov Inst. Math. 1971. MR0340178 (49:4934)

[3] Euler products that correspond to Siegel's modular forms of genus 2, Uspekhi Mat. Nauk 29 (1974), no. 3, 43-110; English transl. in Russian Math. Surveys 29 (1974), no. 3. MR0432552 $(55: 5540)$

[4] , Quadratic forms and Hecke operators, Grundlehren Math. Wiss., Bd. 286, Springer-Verlag, Berlin, 1987. MR 0884891 (88g:11028)

[5] A. O. L. Atkin and J. Lehner, Hecke operators on $\Gamma_{0}(m)$, Math. Ann. 185 (1970), 134-160. MR0268123 (42:3022) 
[6] A. Ogg, Modular forms and Dirichlet series, W. A. Benjamin, Inc., New York-Amsterdam, 1969. MR0256993 (41:1648)

[7] G. Shimura, On modular correspondences for $S p(n, \mathbb{Z})$ and their congruence relations, Proc. Nat. Acad. Sci. U.S.A. 49 (1963), no. 6, 824-828. MR0157009 (28:250)

St. Petersburg Branch, Steklov Mathematical Institute, Russian Academy of Sciences, Fontanka 27, 191023 St. Petersburg, Russia

E-mail address: anandr@pdmi.ras.ru

E-mail address: anatoli.andrianov@gmail.com

Received 2/JUN/2008

Translated by THE AUTHOR 